\title{
A versatile UHV transport and measurement chamber for neutron reflectometry under UHV conditions
}

\author{
A. Syed Mohd, ${ }^{1, *}$ S. Pütter ${ }^{1}, \dagger$ S. Mattauch, ${ }^{1}$ A. Koutsioubas, ${ }^{1}$ H. Schneider, ${ }^{1}$ A. Weber,${ }^{1}$ and T. Brückel ${ }^{1,2}$ \\ ${ }^{1}$ Jülich Centre for Neutron Science (JCNS) at Heinz Maier-Leibnitz Zentrum (MLZ) \\ Forschungszentrum Jülich GmbH, Lichtenbergstr. 1, 85747 Garching, Germany \\ ${ }^{2}$ Jülich Centre for Neutron Science (JCNS) and Peter Grünberg Institute (PGI); JCNS-2, \\ PGI-4: Scattering Methods Forschungszentrum Jülich GmbH, 52425 Jülich, Germany
}

(Dated: December 29, 2016)

\begin{abstract}
We report on a versatile mini ultra high vacuum (UHV) chamber which is designed to be used on the MAgnetic Reflectometer with high Incident Angle (MARIA) of the Jülich Centre for Neutron Science at Heinz Maier-Leibnitz Zentrum in Garching, Germany. Samples are prepared in the adjacent thin film laboratory by molecular beam epitaxy and moved into the compact chamber for transfer without exposure to ambient air. The chamber is based on DN $40 \mathrm{CF}$ flanges and equipped with sapphire view ports, a small getter pump and a wobble stick, which serves also as sample holder.

Here, we present polarized neutron reflectivity measurements which have been performed on Co thin films at room temperature in UHV and in ambient air in a magnetic field of $200 \mathrm{mT}$ and in the Q-range of $0.18 \AA^{-1}$. The results confirm that the Co film is not contaminated during the polarized neutron reflectivity measurement. Herewith it is demonstrated that the mini UHV transport chamber also works as measurement chamber which opens new possibilities for polarized neutron measurements under UHV conditions.
\end{abstract}

\section{INTRODUCTION}

The investigation of thin film samples which are sensitive to ambient air with instruments at large scale facilities is challenging. While simple sputter chambers for thin film growth and in-situ measurements have been realized, complex and much more powerful ultra high vacuum (UHV) systems, e.g. for molecular beam epitaxy (MBE) with several elements cannot be placed on-site due to limited space because the movement of the detector has to be taken into account. Further restrictions appear for neutron reflectivity measurements as a magnet for the magnetic field is required and the sample has to be in the UHV chamber as well as in the center of the yoke of the magnet.

Only few growth chambers are reported for neutron experiments.[1-3] As an alternative approach, ultra-thin films which are sensitive to ambient air are subsequently covered by protective cap layers after fabrication. However, this may change the physical properties of the thin film. [4, 5] Another solution is the installation of a small UHV chamber only dedicated to measurements at the instrument.[6] Consequently, a UHV transfer chamber is required for bridging the gap between the UHV preparation system and the measurement chamber.[7-10] However, this method requires two transfers from one UHV chamber to the other which is inconvenient and time consuming.

We developed a mini UHV chamber made from standard UHV parts which solves these problems. The cham-

\footnotetext{
*Electronic mail:A.Syed.Mohd@fz-juelich.de

$\dagger$ Electronic mail:S.Puetter@fz-juelich.de
}

ber is capable of both, sample transfer and neutron reflectivity measurement under UHV conditions. An excellent candidate for testing the potential of the UHV chamber is the neutron reflectometer MARIA at MLZ in Garching, Germany designed for the investigation of ultra-thin magnetic films.[11]

In this paper, we show the versatility of this chamber. As test sample a $30 \AA$ Co film on $\mathrm{Pt}(205 \AA) / \mathrm{MgO}(001)$ was produced in the MBE system located in an adjacent building to the neutron reflectometer's location. Co was chosen due to its high reactivity with air. We present the first reflectometry measurements under UHV conditions at MARIA. The results reveal also the feasibility of the mini UHV transport chamber as a measurement chamber for neutron reflectometry.

\section{EXPERIMENTAL}

UHV substrate preparation and thin film growth were performed in a DCA M600 MBE system with a base pressure of $10^{-10}$ mbar. A $1 \times 1 \mathrm{~cm}^{2} \mathrm{MgO}(001)$ single crystal (Crystec $\mathrm{GmbH}$, Berlin, Germany) served as substrate. To get rid of the $\mathrm{H}_{2} \mathrm{O}$ at the surface, the $\mathrm{MgO}(001)$ was annealed at $200^{\circ} \mathrm{C}$ under UHV conditions for 2 hours. Subsequently, a $205 \AA \mathrm{Pt}$ buffer layer was deposited at $70^{\circ} \mathrm{C}$ followed by growth of $30 \AA$ Co at $50^{\circ} \mathrm{C}$. The $\mathrm{Pt}$ buffer prevents the Co film from oxidation and intermixing with $\mathrm{MgO}$.[12] The Pt was grown using e-beam evaporation at a rate of $3 \AA / \mathrm{min}$. The deposition rate of Co was kept at $1.2 \AA / \mathrm{min}$ using a high temperature effusion cell. During growth the pressure did not exceed $4 \cdot 10^{-10}$ mbar. The evaporation rate was monitored using a precalibrated quartz microbalance.

The MAgnetic Reflectometer with high Incident An- 


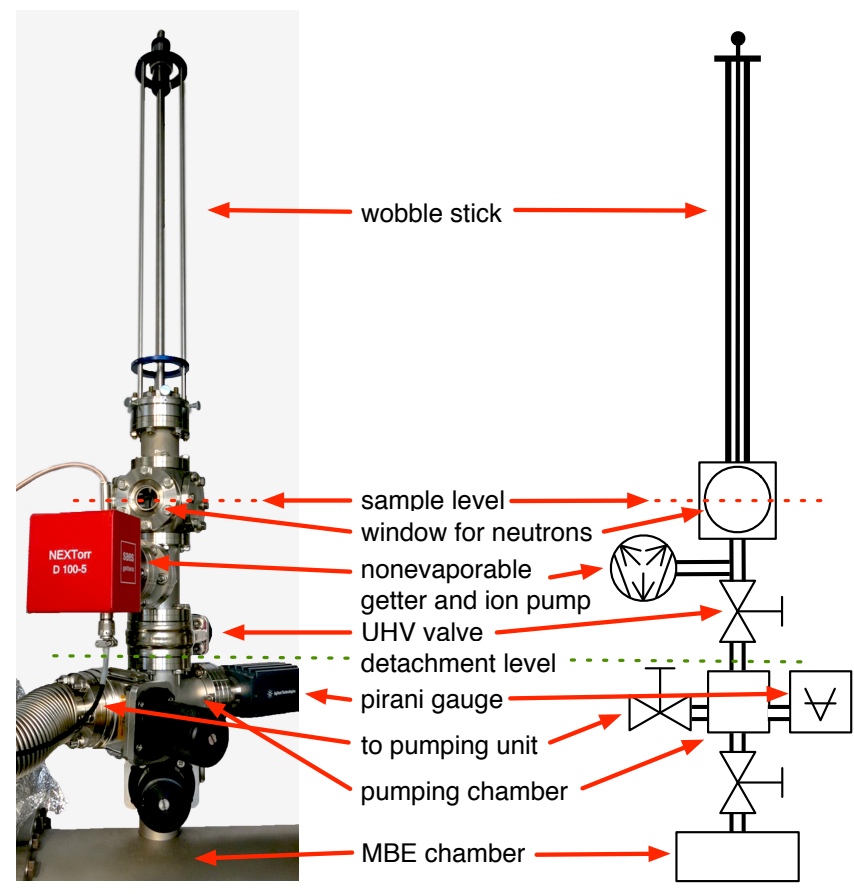

FIG. 1: (Color online) Photo (left) and sketch (right) of the transfer chamber attached to the MBE setup.

gle (MARIA) of the Jülich Centre for Neutron Science at Heinz Maier-Leibnitz Zentrum in Garching, Germany, has a horizontal scattering plane. It provides polarized neutron reflectometry (PNR) in standard operation. At the sample position, a hexapod with a Bruker electromagnet is installed. In-plane magnetic fields of maximum $1.2 \mathrm{~T}$ can be applied. For the transfer chamber the pole shoes have to be unmounted which reduces the magnetic field to max. $600 \mathrm{mT}$ at a gap of about $12 \mathrm{~cm}$. The measurements are performed at room temperature. For data treatment of the PNR measurement the program GenX is used.[13]

\section{SYSTEM LAYOUT}

A photograph and a sketch of the transfer chamber are shown in Fig. 1. The transportable chamber is lightweight (about $10 \mathrm{~kg}$ ) and easy to transport. Special attention has been paid to the compatibility of the chamber to neutron reflectivity measurements. Mostly standard components with DN 40 CF (CF ConFlat, a registered trademark of Varian, Inc.) have been chosen in preferably non-ferromagnetic version. The core part is a DN $40 \mathrm{CF}$ cube made of low magnetic permeability steal (316LN). Two opposing DN 40 CF sapphire view ports are mounted for enabling a clear neutron beam path. The maximum angle of incidence is $13^{\circ}$ for the neutron beam which is sufficient for measurements up to $Q_{z}=0.3 \AA^{-1}$ at $\lambda=4.5 \AA$. Here $Q_{z}$ denotes the component of the scattering vector perpendicular to the sample

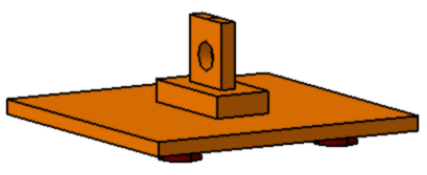

FIG. 2: (Color online) Sketch of the sample holder $\left(2 \times 2 \mathrm{~cm}^{2}\right)$ with small handle on the back. The sample is attached facing downwards.

surface. To reach UHV the transport chamber is baked at $150^{\circ} \mathrm{C}$ for 48 hours.

For easy handling, the sample is attached to a sample holder which is sketched in Fig. 2. On its front side the sample is clamped with bolt heads. On the back side the sample holder has a small handle for being grabbed. The typical sample size is $1 \times 1 \mathrm{~cm}^{2}$. Our sample holder is made from $\mathrm{Cu}$. However, any UHV compatible material is possible.

For inserting the sample holder with the attached sample from the MBE setup into the transfer chamber a wobble stick (Ferrovac GmbH) is used. In general, a wobble stick serves for seizing things and translating and rotating them inside a UHV chamber. Here, together with the sample holder, it also serves for keeping the sample in position for measurement after retracting it into the transfer chamber. The wobble stick can be fixed mechanically from outside to prevent any sample movement. For the given orientation of the transfer chamber in Fig. 1 the sample is facing downwards.

Opposite to the wobble stick a DN $40 \mathrm{CF}$ UHV tee is attached. At 90 degrees from it, a getter pump type Nextorr D 100-5 (SAES Getters SpA, Italy) is installed. The pump is a combination of a sputter ion pump (SIP) and NEG material (nonevaporable getter material) [14] with a maximum pumping speed of $140 \mathrm{l} / \mathrm{s}$. The pump is encased by a $\mu$-metal shield to minimize the magnetic stray field, which is about $0.08 \mathrm{mT}$ at the sample position. This value is well below the remanence of the magnet of the neutron reflectometer and the guide field of $0.2 \mathrm{mT}$. Therefore it does not influence the measurements. Two power supplies are used for ion pump operation, one is placed at the MBE system and the other one is located at the neutron reflectometer. During transfer, only the NEG element is pumping. If necessary, permanent operation of the ion pump can be supplied. The pressure is measured by the ion getter pump. The base pressure of the transfer chamber is about $2 \cdot 10^{-10}$ mbar. The chamber is closed by a customized DN $40 \mathrm{CF}$ valve (VAT $\mathrm{GmbH}$ ) which has a small quartz window inside. This window allows for prealignment of the sample inside transfer chamber thereby observing a reflected laser beam, Fig. 3 . 


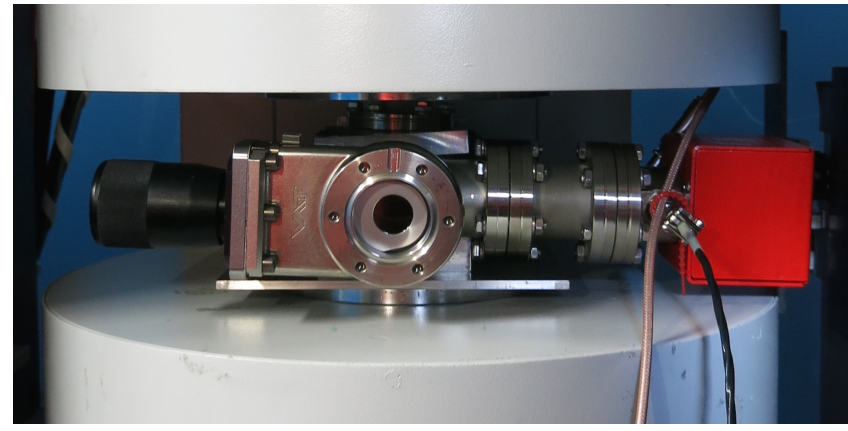

FIG. 3: (Color online) Photo of the transfer chamber inside the MARIA magnet. In the center, the valve with the customized window is visible. On the right the ion getter pump is located.

\section{PROCEDURE OF SAMPLE TRANSFER}

\section{A. Attachment to the MBE setup}

At the MBE setup, the transfer chamber is attached to a DN $40 \mathrm{CF}$ cross with a valve, a Pirani vacuum gauge and a pumping unit, Fig. 1. Within the MBE setup samples are moved horizontally in a trolley, facing downwards. For the sample transfer a trolley is moved below the transfer chamber. A window in the UHV chamber at the level of the trolley enables the control of the transfer. After opening the valves the wobble stick of the transfer chamber is moved downwards to the trolley. The pincer grip of the wobble stick grabs the handle of the sample (Fig. 2) and by withdrawing the wobble stick into the transfer chamber the sample is moved to its position. After closing the valve and venting the UHV cross the transfer chamber can be detached from the MBE system and carried to the neutron instrument.

\section{B. Neutron reflectivity measurements at MARIA}

The transfer chamber is transferred to the neutron instrument within approximately 5 minutes. It is inserted horizontally between the pole shoes of the magnet, Fig. 3 , and the pump is attached to its power supply. The pressure does not change during this procedure. After keeping the transfer chamber without power for 24 hours the pressure rises up to $1.3 \cdot 10^{-9}$ mbar. For sensitive samples constant pumping is enabled. The PNR measurements are performed at room temperature. A typical measurement of spin up $(\mathrm{R}+)$ and spin down (R-) reflectivity usually takes three hours.

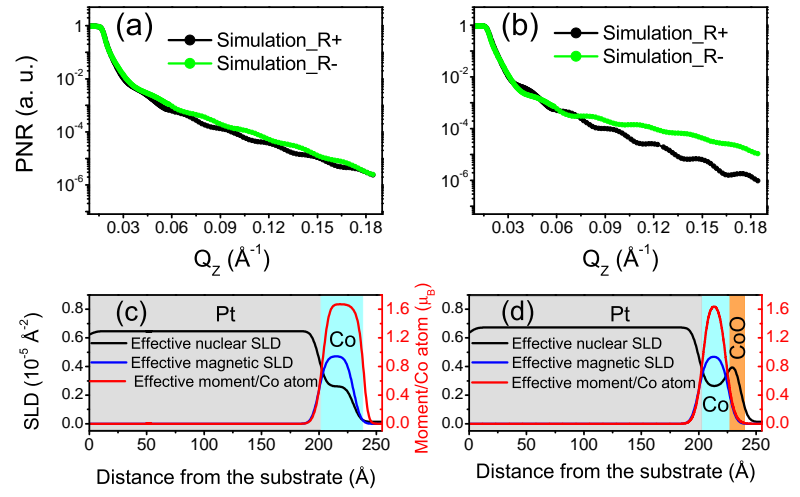

FIG. 4: (Color online) Simulated PNR curves of (a) Co film: $30 \AA \mathrm{Co} / 205 \AA \mathrm{Pt} / \mathrm{MgO}(001)$ and (b) a partially oxidized Co film: $10 \AA \mathrm{CoO} / 25 \AA \mathrm{Co} / 205 \AA \mathrm{Pt} / \mathrm{MgO}(001)$. In (c) and (d) the nuclear and magnetic SLD profiles of the reflectivity curves in (a) and (b) as well as the effective moment per Co atom are shown.

\section{RESULTS AND DISCUSSION}

\section{A. PNR-Measurements}

In this part, we present polarized neutron reflectivity (PNR) measurements of a $30 \AA$ Co film which was first prepared in the MBE setup and then measured in the transport chamber under UHV conditions at MARIA. Afterwards the same sample was measured again in ambient conditions after taking it out of the transfer chamber.

For comparison, we first discuss simulated PNR curves of $30 \AA \quad \mathrm{Co} / 205 \quad \AA \quad \mathrm{Pt} / \mathrm{MgO}(001)$ and $10 \AA \mathrm{CoO} / 25 \AA \mathrm{Co} / 205 \AA \mathrm{Pt} / \mathrm{MgO}(001)$ in Figs. 4(a) and (b), respectively. While the nuclear Scattering Length Density (nuclear SLD) is always the same the magnetic Scattering Length Density (magnetic SLD) depends on the in-plane magnetization of the sample parallel or anti-parallel to the applied field. As a result separate spin up $(\mathrm{R}+)$ and the spin down $(\mathrm{R}-)$ channels give information about the magnetic profile of the film. In the simulation with GenX [13] we utilized the nominal nuclear and magnetic scattering length density of $\mathrm{MgO}$, $\mathrm{Pt}$, $\mathrm{Co}$ and $\mathrm{CoO}$, respectively. The film roughness was set to a typical value of $5 \AA$ at each interface.

The resulting effective nuclear and magnetic SLD depth profiles as well as the effective magnetic moment per Co atom are shown in Figs. 4(c) and (d), respectively. In PNR, roughness at the interface is reflected as a continuous variation of the SLD across the interface. As a consequence, the SLDs and the effective magnetic moment exceed the nominal film thicknesses given by the coloured background as guide to the eye in the figures. 


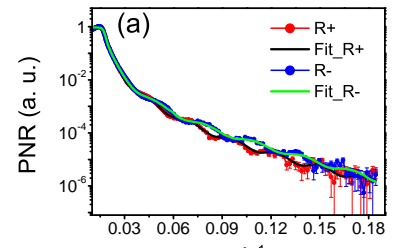

$Q_{Z}\left(\AA^{-1}\right)$

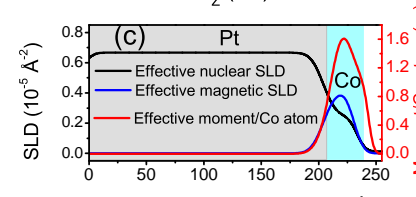

Distance from the substrate $(\AA)$

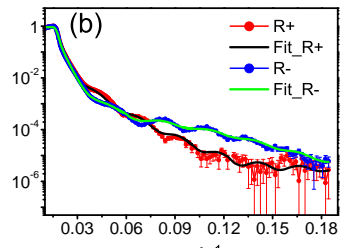

$Q_{Z}\left(\AA^{-1}\right)$

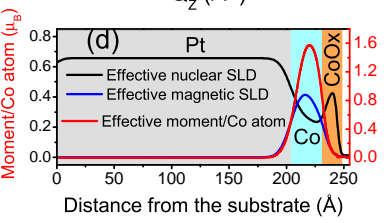

FIG. 5: (Color online) PNR curves of the $30 \AA$ Co film (a) measured under UHV conditions utilizing the UHV transport chamber and (b) measured after exposing the same Co film to ambient air for 6 hours. In (c) and (d) the neutron SLD profiles obtained from the fitting of the PNR data measured under UHV conditions and in ambient air are shown.

For the profile of the effective magnetic moment per Co atom, we have normalized the magnetic SLD with the nuclear SLD utilizing the formula of Ankner et al. [15]:

$$
\frac{\text { Magnetic moment }}{\text { atom }}=\frac{b}{c} \cdot \frac{\text { Magnetic SLD }}{\text { Nuclear SLD }}
$$

where $b$ is the scattering length of the atom and $c=$ $2.69 \cdot 10^{-5} \AA / \mu_{\mathrm{B}}$. The simulation in Fig. 4 clearly shows that the PNR measurement of an oxidized Co film will differ from that of a pure Co film.

In Fig. 5 we present PNR data of $\mathrm{Co} / \mathrm{Pt} / \mathrm{MgO}(001)$ measured first in UHV utilizing the transport chamber and second ex-situ after exposing it to ambient air for about 6 hours, see Fig. 5(a) and (b), respectively. The pressure in the transport chamber during the measurement in UHV was $4 \cdot 10^{-10}$ mbar. The measurements were performed at room temperature with a magnetic field of $200 \mathrm{mT}$, which is well above the saturation field of Co thin films of $10 \mathrm{mT}[16,17]$. The direction of the magnetic field was kept parallel to the polarisation axis of the neutron beam. Already a qualitative comparison of Fig. 5(a) and (b) reveals differences in the reflectivity curves which are in good accordance with the ones in the simulated curves, Fig. 4.

For further insight, the UHV and ex-situ PNR data were fitted, for the resulting parameters see Table I. For all fitting parameters, the error was found to be $\pm 1 \AA$. For the ex-situ measured PNR data an additional Co oxide layer $\left(\mathrm{CoO}_{x}\right)$ on top of the Co layer had to be taken into account. It is well known that Co quickly oxidizes in air.

Figs. 5(c) and (d) show the depth profiles of the effective nuclear SLD, effective magnetic SLD and effective magnetic moment per Co atom obtained from the fitting. In order to gain higher accurracy in the magnetic

\begin{tabular}{|l|r|r|r|r|}
\hline & \multicolumn{2}{|l|}{$\begin{array}{l}\text { in UHV } \\
\left(4 \cdot 10^{-10} \text { mbar }\right)\end{array}$} & \multicolumn{2}{l|}{$\begin{array}{l}\text { ex } \begin{array}{l}\text { (aftu } \\
\text { in ambient } \text { hours }\end{array} \\
\text { in air }\end{array}$} \\
\hline Layer & $t(\AA)$ & $\sigma(\AA)$ & $t(\AA)$ & $\sigma(\AA)$ \\
\hline $\mathrm{CoO}_{x}$ & - & - & 9 & 4 \\
\hline $\mathrm{Co}$ & 30 & 6 & 26 & 6 \\
\hline $\mathrm{Pt}$ & 205 & 7 & 205 & 7 \\
\hline
\end{tabular}

TABLE I: Thickness $(t)$ and roughness $(\sigma)$ of the $\mathrm{CO}_{x}$, Co (sum of $\mathrm{Co}_{1}, \mathrm{Co}_{2}$ and $\mathrm{Co}_{3}$ layer) and $\mathrm{Pt}$ layers of the same sample measured using PNR measurements in UHV and exsitu, respectively. The error in each value is $\pm 1 \AA$.

profile, the Co film was subdivided into three layers $\left(\mathrm{Co}_{1}\right.$, $\mathrm{Co}_{2}$ and $\mathrm{Co}_{3}$ ) of $10 \AA$ thickness. We allowed the nuclear and magnetic SLD of layer $\mathrm{Co}_{1}$ (in contact with vacuum or air) and layer $\mathrm{Co}_{3}$ (in contact with the $\mathrm{Pt}$ film) to vary from its nominal value while for layer $\mathrm{Co}_{2}$ (middle part of Co film) the SLDs were fixed to their nominal values.

In total, we may conclude that the UHV transfer chamber is fully working as the transferred Co thin film is not oxidized during its measurement at MARIA.

\section{SUMMARY AND CONCLUSION}

In this paper we present the setup and applicability of a versatile transfer chamber for measuring polarized neutron reflectometry under UHV conditions. The setup is described in detail, being assembled by standard DN $40 \mathrm{CF}$ UHV parts. Only one transfer is necessary from sample fabrication to PNR measurement which may be performed back and forth. Hence, also PNR studies of the same thin film with increasing thickness or of multilayer samples are possible.

The polarized neutron reflectometry measurements were performed at the neutron reflectometer MARIA on $30 \AA$ Co thin film grown on $205 \AA \mathrm{Pt} / \mathrm{MgO}(001)$ of $1 \times 1 \mathrm{~cm}^{2}$ and transferred to MARIA under UHV conditions. The evaluation of the data reveals no oxidation of the Co film, showing the stability of this chamber for PNR measurements under UHV conditions.

The general setup of the transfer chamber is versatile and may easily be adopted to other large scale instruments.

\section{Acknowledgments}

Technical help of S. Staringer is gratefully acknowledged. This project is part of the nanoscience foundry and fine analysis project (NFFA)[18] and has received funding from the EU's H2020 research and innovation programme under grant agreement n. 654360. 
[1] T. Nawrath, H. Fritzsche, F. Klose, J. Nowikow, and H. Maletta, Phys. Rev. B 60, 9525 (1999).

[2] J. A. Dura and J. LaRock, Rev. Sci. Instrum. 80, 073906 (2009).

[3] W. Kreuzpaintner, B. Wiedemann, S. Mayr, A. Paul, T. Mairoser, A. Schmehl, A. Herrnberger, J. Stahn, J.-F. Moulin, K. Panagiotis, et al., in 13th Surface X-Ray and Neutron Scattering Conference (2014).

[4] J. F. Calleja, M. C. Contreras, R. Matarranz, E. Navarro, Y. Huttel, A. Cebollada, and G. Armelles, J. Appl. Phys. 97, 104302 (2005).

[5] Y. Huttel, E. Navarro, N. D. Telling, G. van der Laan, F. Pigazo, F. J. Palomares, C. Quintana, E. Roman, G. Armelles, and A. Cebollada, Phys. Rev. B 78, 104403 (2008).

[6] T. Slobodskyy, P. Schroth, D. Grigoriev, A. A. Minkevich, D. Z. Hu, D. M. Schaadt, and T. Baumbach, Rev. Sci. Instrum. 83, 105112 (2012).

[7] P. Jiřiček, M. Cukr, V. Kolar̆ik, and S. Koc, Rev. Sci. Instrum. 69, 2804 (1998).

[8] G. Firpo, F. Buatier de Mongeot, C. Boragno, and U. Valbusa, Rev. Sci. Instrum. 76, 026108 (2005).

[9] F. U. Renner, Y. Gründer, and J. Zegenhagen, Rev. Sci. Instrum. 78, 033903 (2007).
[10] Y. Watanabe, Y. F. Nishimura, R. Suzuki, H. Uehara, T. Nimura, A. Beniya, N. Isomura, K. Asakura, and S. Takakusagi, J. Vac. Sci. \& Techn. A 34, 023201 (2016).

[11] S. Mattauch, A. Koutsioubas, and S. Pütter, J. Large-Scale Research Facilities 1, A8 (2015), http://dx.doi.org/10.17815/jlsrf-1-29.

[12] J.-B. Laloé, A. Ionescu, S. Easton, N.-J. Steinke, T. J. Hayward, H. Kurebayashi, J. A. C. Bland, T. R. Charlton, R. M. Dalgliesh, and S. Langridge, Appl. Phys. Lett. 93, 012505 (2008).

[13] M. Björck and G. Andersson, J. Appl. Crystallogr. 40, 1174 (2007).

[14] C. D. Park, S. M. Chung, and P. Manini, J. Vac. Sci. \& Techn. A 29, 011012 (2011).

[15] J. F. Ankner, C. F. Majkrzak, and H. Homma, Journal of Applied Physics 73, 6436 (1993).

[16] J. A. C. Bland, R. D. Bateson, P. C. Riedi, R. G. Graham, H. J. Lauter, J. Penfold, and C. Shackleton, Journal of Applied Physics 69, 4989 (1991).

[17] S. Pütter, H. F. Ding, Y. T. Millev, H. P. Oepen, and J. Kirschner, Phys. Rev. B 64, 092409 (2001).

[18] See http://www.nffa.eu for futher information on the nanoscience foundry and fine analysis project. 